\title{
FORMAL DIFFERENTIAL GEOMETRY AND NAMBU-TAKHTAJAN ALGEBRA
}

\author{
YURI L. DALETSKII \\ National Technical University of Ukraine \\ 37 Peremogy prosp., Kiev, Ukraine \\ E-mail: dalet@stoch.freenet.kiev.ua \\ VITALY A. KUSHNIREVITCH \\ National Technical University of Ukraine \\ 37 Peremogy prosp., Kiev, Ukraine
}

1. Introduction. In this paper we consider a formal algebraic construction such that an algebraic apparatus of differential geometry could be interpreted as its realization: construction of $(\mathfrak{A}, \mathfrak{D})$-systems. This construction was offered by I. M. Gelfand and one of the authors (see $[1,2]$ ) as a basis of non-commutative differential geometry in the following sense: we replace an algebra of functions on some manifold by a module over a Lie algebra.

Thus, we take a pair $(M, \mathfrak{A})$ consisting of Lie algebra $\mathfrak{A}$ and $\mathfrak{A}$-module $M$ as an abstract non-commutative analog of a smooth manifold (see [3,4]).

This approach allows us to extend a stock of realizations of our theory. Among them there are a formal calculus of variations (see [3,4]) and also a construction with a matrix algebra as $M$ close to [5]. In [2,6,7] an application of formal apparatus mentioned to the situation, when a Lie algebra of Hochschild cohomology acts in a complex $(\Omega, d)$ of Hochschild homology with cyclic differential is considered. The results of [8] about Poisson brackets on differential forms and multivector fields on a smooth manifold are also described and generalized in the frameworks of the formal construction mentioned (see also our preprint [9]). Construction of a many-linear analog of Lie algebra introduced by Y. Nambu and developed by L. Takhtajan (see $[10,11,12]$ ) also is connected with our approach.

1991 Mathematics Subject Classification: 17B70, 17A42.

The research described in this publication was made possible in part by Grant No U44200 from the International Science Foundation.

This work was supported by the Fundamental Research Foundation of the State Committee on Science and Technology of Ukraine.

The paper is in final form and no version of it will be published elsewhere. 
Following $[1,2]$ this paper is written in terms of superalgebra, which is necessary in essence. Besides that it leads to the further extension of applications including, for instance, an apparatus of differential geometry of supermanifolds and also graded formal calculus of variations (see $[13,14]$ ).

Note that different aspects of non-commutative differential geometry in wide sense are elaborated by A. Connes, D. Kastler, S. Woronowicz and their followers (see $[15,16]$ and references there).

2. Main notation. A linear space $(\mathfrak{L}, p)$ is a graded linear space with a parity function $p$. Values of $p$ are integer numbers or integer vectors. In the last case we understand $p \cdot p^{\prime}=\sum_{k=1}^{m} \lambda_{k} \lambda_{k}^{\prime}$ as a product of $p=\left(\lambda_{1}, \ldots, \lambda_{m}\right)$ and $p^{\prime}=\left(\lambda_{1}^{\prime}, \ldots, \lambda_{m}^{\prime}\right)$. An element $x \in \mathfrak{L}$ is said to be even or odd, if the number $p^{2}$ is relatively even or odd. A parity of a map of linear spaces is consistent with elements of these spaces in an ordinary way: $p(f(x))=p(f)+p(x)$.

A Lie algebra $(\mathfrak{A},[\cdot, \cdot], p)$ is a linear space $(\mathfrak{A}, p)$ with a bilinear operation $[\cdot, \cdot]: \mathfrak{A} \times \mathfrak{A} \rightarrow$ $\mathfrak{A}$, which satisfies a condition of skew symmetry

$$
[a, b]=-(-1)^{p(a) p(b)}[b, a]
$$

and Jacobi identity, which one can write in the form

$$
\operatorname{ad}_{a}[b, c]=\left[\operatorname{ad}_{a} b, c\right]+(-1)^{p(a) p(b)}\left[b, \operatorname{ad}_{a} c\right],
$$

where $\operatorname{ad}_{a} b=[a, b]$ is an adjoint action of bracket.

A module $(M, p)$ over a Lie algebra $(\mathfrak{A},[\cdot, \cdot], p)$ is a linear space, on which left action $a \times m \mapsto L_{a} m(a \in \mathfrak{A}, m \in M)$ is defined and the following condition

$$
\left[L_{a}, L_{b}\right]^{\vee} m=L_{[a, b]} m
$$

holds. Here and below we use the following notation: $[A, B]^{\vee}=A B-(-1)^{p(A) p(B)} B A$ for $A$ and $B$ being operators.

A complex $\left(\Omega=\bigoplus_{k=0}^{\infty} \Omega_{k}, d, p\right)$ is described as a linear space $(\mathfrak{L}, \vec{p})$ with new graduation $\vec{p}(\omega)=(p(\omega), k)\left(\omega \in \Omega_{k}\right)$. It is called a complex over Lie algebra $(\mathfrak{A},[\cdot, \cdot], p)$ ( $\mathfrak{A}$-complex), if the actions $i_{a}$ and $L_{a}$ with changed parities $\vec{p}\left(i_{a}\right)=(p(a),-1)$ and $\vec{p}\left(L_{a}\right)=(p(a), 0)$ are defined on $\Omega$ and the following relations

$$
[d, d]^{\vee}=0, \quad\left[i_{a}, i_{b}\right]^{\vee}=0, \quad L_{a}=\left[i_{a}, d\right]^{\vee}, \quad\left[L_{a}, i_{b}\right]^{\vee}=i_{[a, b]}
$$

are taken place. Then any $\Omega_{k}$ is $\mathfrak{A}$-module with the action $L_{a}$ and $\left[L_{a}, L_{b}\right]^{\vee}=L_{[a, b]}$ $(a, b \in \mathfrak{A})$.

Below, where it does not lead to misunderstandings, the sign $p$ will be omitted.

If $M$ is $\mathfrak{A}$-module, then $\mathfrak{A}$-complex $\Omega(M, \mathfrak{A})$ (de Rham complex of a pair $(M, \mathfrak{A})$ ) is defined canonically:

$$
\begin{array}{r}
\Omega_{0}=M, \quad \Omega_{k}=\{\omega \mid \omega: \underbrace{\mathfrak{A} \times \ldots \times \mathfrak{A}}_{k} \rightarrow M \text { is } k \text {-linear skew symmetric map }\} \\
(d \omega)\left(a_{1}, \ldots, a_{n+1}\right)=\sum_{k=1}^{n+1}(-1)^{k+1+p_{k} p(\omega)+p_{k} \Sigma_{r<k} p_{r}} L_{a_{k}}\left(\omega\left(a_{1}, \ldots, \widehat{a}_{k}, \ldots, a_{n+1}\right)\right)+ \\
+\sum_{j<k}(-1)^{j+k+p_{j} \Sigma_{r<j} p_{r}+p_{k} \Sigma_{r<k} p_{r}+p_{j} p_{k}} \omega\left(\left[a_{j}, a_{k}\right], a_{1}, \ldots, \widehat{a}_{j}, \ldots, \widehat{a}_{k}, \ldots, a_{n+1}\right),
\end{array}
$$




$$
\left(i_{a} \omega\right)\left(b_{1}, \ldots, b_{k}\right)=(-1)^{p(\omega) p(a)} \omega\left(a, b_{1}, \ldots, b_{k}\right)
$$

(Sign $\widehat{a}$ means that $a$ is omitted.)

We consider the following generalization of $\mathfrak{A}$-module and $\mathfrak{A}$-complex. Suppose Lie algebra structures $[\cdot, \cdot]^{\xi}$ and actions $L_{a}^{\xi}$ linearly depend on parameter $\xi$ valued in some linear space $\mathfrak{L}$; and $(2.1)$ is valid for any $\xi \in \mathfrak{L}$. Then we say that $M$ is multimodule over $\mathfrak{A}$. In this case brackets are bound by the following relation

$$
\begin{aligned}
{\left[\left[a_{1}, a_{2}\right]^{\xi_{1}}, a_{3}\right]^{\xi_{2}}+\left[\left[a_{1}, a_{2}\right]^{\xi_{2}}, a_{3}\right]^{\xi_{1}}+(-1)^{p_{1} p_{2}}\left[a_{2},\left[a_{1}, a_{3}\right]^{\xi_{1}}\right]^{\xi_{2}}+} \\
+(-1)^{p_{1} p_{2}}\left[a_{2},\left[a_{1}, a_{3}\right]^{\xi_{2}}\right]^{\xi_{1}}=\left[a_{1},\left[a_{2}, a_{3}\right]^{\xi_{1}}\right]^{\xi_{2}}+\left[a_{1},\left[a_{2}, a_{3}\right]^{\xi_{2}}\right]^{\xi_{1}} .
\end{aligned}
$$

Proposition 2.1. Let $M$ be a multimodule over $\mathfrak{A}^{\xi}=\left(\mathfrak{A},[\cdot, \cdot]^{\xi}\right)$ and for any $\xi \in$ $\mathfrak{L}\left(\Omega\left(M, \mathfrak{A}^{\xi}\right), d^{\xi}\right)$ be the set of corresponding de Rham complexes. Then the following relations: $d^{\xi_{1}+\xi_{2}}=d^{\xi_{1}}+d^{\xi_{2}}$ and $\left[d^{\xi_{1}}, d^{\xi_{2}}\right]=0$ hold.

We say that $\left(\Omega, d^{\xi}\right)$ is a multicomplex.

3. Brackets generated by differentials. Let $\mathfrak{G}=(\mathfrak{G},[\cdot, \cdot], p)$ be a Lie algebra. Any odd element $d \in \mathfrak{G}$ is called a differential in $\mathfrak{G}$, if the following property

$$
[d, d]=0
$$

holds.

With any differential we associate a Lie map

$$
L^{d}: g \mapsto[g, d]
$$

and a $d$-bracket: bilinear operation

$$
\left[g_{1}, g_{2}\right]_{d}=\left[L^{d} g_{1}, g_{2}\right]=\left[\left[g_{1}, d\right], g_{2}\right] .
$$

It easily follows from the Jacobi identity that

$$
L^{d}\left[g_{1}, g_{2}\right]_{d}=\left[L^{d} g_{1}, L^{d} g_{2}\right] \quad\left(g_{1}, g_{2} \in \mathfrak{G}\right)
$$

Let $\pi=p(d), \widetilde{p}(g)=p(g)+\pi$.

Proposition 3.1. For any $g_{j} \in \mathfrak{G}\left(p_{j}=p\left(g_{j}\right), \widetilde{p}_{j}=p_{j}+\pi\right)$ the following relations hold

$$
\begin{gathered}
{\left[g_{1}, g_{2}\right]_{d}+(-1)^{\widetilde{p}_{1} \widetilde{p}_{2}}\left[g_{2}, g_{1}\right]_{d}=L^{d}\left((-1)^{\pi p_{2}}\left[g_{1}, g_{2}\right]\right)} \\
(-1)^{\widetilde{p}_{1} \widetilde{p}_{3}}\left[\left[g_{1}, g_{2}\right]_{d}, g_{3}\right]_{d}+\mathfrak{y}=\frac{1}{3} L^{d}\left\{(-1)^{p_{1} \widetilde{p}_{3}}\left(\left[g_{1},\left[g_{2}, g_{3}\right]\right]_{d}-2\left[\left[g_{1}, g_{2}\right]_{d}, g_{3}\right]\right)+\mathfrak{y}\right\},
\end{gathered}
$$

(by the sign $\mathfrak{y}$ we denote terms which are obtained from the latter one by the cyclic permutation of indexes).

Consider now an even element $\Theta$. Let $d_{\Theta}=[\Theta, d]$. We have

$$
\left[d_{\Theta}, d\right]=0, \quad\left[d_{\Theta}, d_{\Theta}\right]=\left[[\Theta, \Theta]_{d}, d\right]
$$

Then $\Theta$ is called a conditional differential, if $d_{\Theta}$ is a differential: $\left[d_{\Theta}, d_{\Theta}\right]=0$. The sufficient condition of $\Theta$ being conditional differential is

$$
[\Theta, \Theta]_{d}=0 .
$$




\section{Structures on commutative Lie subalgebras}

Definition. A commutative subalgebra $\mathfrak{L}$, which is invariant with respect to $d$ bracket is called a $d$-space in $\mathfrak{G}$ :

$$
[\mathfrak{L}, \mathfrak{L}]=0, \quad[\mathfrak{L}, \mathfrak{L}]_{d} \subset \mathfrak{L} .
$$

We get from from (3.1)-(3.3) the following theorem.

THEOREM 4.1. d-bracket induces a Lie algebra structure $\mathfrak{L}_{d}=\left(\mathfrak{L},[\cdot, \cdot]_{d}, \widetilde{p}\right)$ in a d-space $\mathfrak{L}$. The Lie map $L:\left(\mathfrak{L},[\cdot, \cdot]_{d}, \widetilde{p}\right) \rightarrow(\mathfrak{L},[\cdot, \cdot], p)$ is a homomorphism of Lie algebras.

Let $\mathfrak{G}$ be a Lie algebra and let $\Omega$ be a commutative Lie subalgebra, which is invariant with respect to Lie map:

$$
[\Omega, \Omega]=0, \quad[\Omega, d] \subset \Omega .
$$

Then it is evident that $\Omega$ is a $d$-comutative $d$-space (i. e. $[\Omega, \Omega]_{d}=0$ ).

THEOREM 4.2. Let $\Omega$ obey the condition (4.1), and $\Theta$ be a conditional differential, and the following condition holds

$$
\omega_{1}, \omega_{2} \in \Omega \Rightarrow\left[\left[\omega_{1}, \Theta\right], \omega_{2}\right]=0 .
$$

Then $\Omega$ is a $d_{\Theta}$-space possessing Lie algebra structure $\Omega_{d_{\Theta}}=\left(\Omega,[\cdot, \cdot]_{d_{\Theta}}, \widetilde{p}\right)$ and

$$
L^{d_{\Theta}}:\left[\omega_{1}, \omega_{2}\right]_{d_{\Theta}} \mapsto\left[\left[\omega_{1}, d_{\Theta}\right],\left[\omega_{2}, d_{\Theta}\right]\right]
$$

is a homomorphism of Lie algebras $\Omega_{d_{\Theta}} \rightarrow \mathfrak{G}$.

5. Structures on quotient space. Consider a kernel and an image of Lie map $L^{d}$ :

$$
\begin{gathered}
\mathcal{Z}_{d}=\{z \in \mathfrak{G} \mid[z, d]=0\} \\
\mathcal{J}_{d}=\{h=[g, d] \mid g \in \mathfrak{G}\} \subset \mathcal{Z}_{d}
\end{gathered}
$$

TheOREm 5.1. The linear space $\mathcal{Z}_{d} \subset \mathfrak{G}$ is an ideal with respect to $[\cdot, \cdot]_{d}$. A quotient space $\mathcal{K}_{d}=\mathfrak{G} / \mathcal{Z}_{d}$ possesses a Lie algebra structure $\left(\mathcal{K}_{d},[\cdot, \cdot]_{d}, \widetilde{p}\right)$, and the Lie map

$$
L^{d}:\left(\mathcal{K}_{d},[\cdot, \cdot]_{d}, \widetilde{p}\right) \rightarrow\left(\mathcal{J}_{d},[\cdot, \cdot], p\right)
$$

is an isomorphism of Lie algebras.

Let now $\Theta$ be a conditional differential. Then due to (3.4) $\Theta$ is a differential in $\mathcal{K}_{d}$. Introduce the bracket

$$
\llbracket g_{1}, g_{2} \rrbracket_{\Theta}=\left[\left[g_{1}, \Theta\right]_{d}, g_{2}\right]_{d} .
$$

TheOrem 5.2. A space $\Omega / \mathcal{Z}_{d}$ is a $\Theta$-space in $\mathcal{K}_{d}$. Therefore $\left(\Omega / \mathcal{Z}_{d}, \llbracket \cdot, \cdot \rrbracket_{\Theta}, p\right)$ is a Lie algebra, and a Lie map

$$
\mathfrak{L}^{\Theta}: \omega \mapsto[\omega, \Theta]_{d}=[[\omega, d], \Theta]
$$

is a homomorphism of Lie algebras $\Omega / \mathcal{Z}_{d} \rightarrow \mathcal{K}_{d}$.

6. ( $\mathfrak{A}, \mathfrak{D})$-structures and modules. An $(\mathfrak{A}, \mathfrak{D})$-structure is a Lie algebra $\mathfrak{G}=$ $\mathfrak{G}(\mathfrak{A}, \mathfrak{D})$ generated by commutative Lie subalgebras $\mathfrak{A}$ and $\mathfrak{D}$, and the following conditions hold: 
(i) $\mathfrak{D}$ consists of differentials;

(ii) $\mathfrak{A}$ is a $\mathfrak{D}$-space (i. e. $\forall d \in \mathfrak{D} \mathfrak{A}$ is a $d$-space):

$$
[[\mathfrak{A}, \mathfrak{D}], \mathfrak{A}] \subset \mathfrak{A}
$$

Under these conditions a $\mathfrak{G}$-module is called $(\mathfrak{A}, \mathfrak{D})$-module.

Let $(\Omega, \mathfrak{D})$ be a multicomplex over $\mathfrak{A}$. Then $\Omega$ is an $(\mathfrak{A}, \mathfrak{D})$-module. In particular, multimodule $(M, \mathfrak{A})$ generates the multicomplex $\Omega(M, \mathfrak{A})$ and therefore the $(\mathfrak{A}, \mathfrak{D})$-module.

On the contrary

Proposition 6.1. If $\Omega$ is a ( $\mathfrak{A}, \mathfrak{D})$-module, then any differential $d \in \mathfrak{D}$ generates a Lie algebra structure $\left(\mathfrak{A},[\cdot, \cdot]_{d}, p+\pi\right)$ in $\mathfrak{A}$. Moreover, $\Omega$ becomes a multimodule with an action $L_{a}^{d} \omega=[a, d] \omega$.

R e mark. If $\Omega=\Omega(M, \mathfrak{A})$ is the de Rham complex of a pair $(M, \mathfrak{A})$, then Lie algebra structure obtained in $\mathfrak{A}$ coincides with initial one.

Consider $(\mathfrak{A}, \mathfrak{D})$-module $\Omega$. Denote by $(\Omega \widetilde{\mathfrak{A}}, \mathfrak{D})$ a corresponding Lie algebra and by $U E A(\Omega, \mathfrak{A}, \mathfrak{D})$ its universal enveloping algebra. Generating by $U E A(\Omega, \mathfrak{A}, \mathfrak{D})$ Lie algebra with the bracket $[\cdot, \cdot]$, which is uniquely defined by Leibnitz rule

$$
[a b, c]=a[b, c]+(-1)^{p(b) p(c)}[a, c] b
$$

is said to be an extended $(\Omega, \mathfrak{A}, \mathfrak{D})$-structure. It contains the commutative Lie subalgebras $\Omega, \mathfrak{A}, \mathfrak{D}$ and together with them the commutative Lie subalgebras $\widetilde{\mathfrak{A}}, \widetilde{\Omega}$, which are obtained from the corresponding symmetric algebras by the bracket $[\cdot, \cdot]$.

Note that a symmetric algebra with a parity $(p, 1)$ is an antisymmetric one with a parity $p$.

In such a situation elements of $\widetilde{\Omega}$ are said to be differential forms, elements of $\mathfrak{A}$ - vector fields, elements of $\widetilde{\mathfrak{A}}$ - multivector fields, elements of $\widetilde{\Omega} \otimes \mathfrak{A}$ - vectorvalued differential forms.

Below we consider a different structures of Lie algebras arising on these spaces in view of sections 4 and 5 .

7. Structures on vector fields and on vectorvalued differential forms. Let $(\Omega, \mathfrak{A}, \mathfrak{D})$ be an extended $(\mathfrak{A}, \mathfrak{D})$-structure. By definition $\mathfrak{A}$ (and therefore $\widetilde{\mathfrak{A}}$ ) is a $d$-space for all $d \in \mathfrak{D}$. Hence, the following result is taken place.

THEOREM 7.1. Bracket

$$
[A, B]_{d}=[[A, d], B]
$$

equips $\widetilde{\mathfrak{A}}$ with a Lie algebra structure $\left(\widetilde{\mathfrak{A}},[\cdot, \cdot]_{d}, p+\pi\right)$. Restriction $\left(\widetilde{\mathfrak{A}},[\cdot, \cdot]_{d}, p+\pi\right) \mid \mathfrak{A}$ coincides with the structure from Proposition 6.1. The following relation holds

$$
\left[A_{1} A_{2}, B\right]_{d}=A_{1}\left[A_{2}, B\right]_{d}+(-1)^{p\left(A_{2}\right) p(B)}\left[A_{1}, B\right] A_{2} .
$$

Consider now vectorvalued forms. 
TheOREM 7.2. A space $\widetilde{\Omega} \otimes \mathfrak{A}$ possesses a Lie algebra structure $\left(\widetilde{\Omega} \otimes \mathfrak{A},[\cdot, \cdot]_{d}^{\prime}, p+\pi\right)$ with the bracket

$$
\begin{array}{r}
{\left[\omega_{1} a_{1}, \omega_{2} a_{2}\right]_{d}^{\prime}=(-1)^{p^{\prime}\left(a_{1}\right) p\left(\omega_{2}\right)} \omega_{1} \omega_{2}\left[a_{1}, a_{2}\right]_{d}+\left[\omega_{1} a_{1}, \omega_{2}\right]_{d} a_{2}-} \\
-(-1)^{p^{\prime}\left(\omega_{1} a_{1}\right) p^{\prime}\left(\omega_{2} a_{2}\right)}\left[\omega_{2} a_{2}, \omega_{1}\right]_{d} a_{1},
\end{array}
$$

where $p^{\prime}(\omega a)=p(\omega)+p^{\prime}(a), p^{\prime}(a)=(\widetilde{p}(a), 0)$.

This fact follows from the general assertion (see [1]).

Theorem 7.3. Let a commutative algebra $M$ be an $\mathfrak{A}$-module and elements of $\mathfrak{A}$ be differentials in $M$. Then $\mathfrak{A}_{M}=M \otimes \mathfrak{A}$ possesses Lie algebra structure with the bracket

$$
\begin{aligned}
& {\left[m_{1} \otimes a_{1}, m_{2} \otimes a_{2}\right]=(-1)^{p\left(a_{1}\right) p\left(m_{2}\right)} m_{1}, m_{2} \otimes\left[a_{1}, a_{2}\right]+} \\
& \quad+m_{1}\left(a_{1} m_{2}\right) \otimes a_{2}-(-1)^{p\left(m_{1} \otimes a_{1}\right) p\left(m_{2} \otimes a_{2}\right)} m_{2}\left(a_{2} m_{1}\right) \otimes a_{1} .
\end{aligned}
$$

In addition, $M$ is $\mathfrak{A}_{M}$-module:

$$
(m \otimes a) m^{\prime}=m\left(a m^{\prime}\right) .
$$

8. Structures on differential forms generating by bivector fields. Let an extended $(\Omega, \mathfrak{A}, \mathfrak{D})$-structure be generated by a de Rham complex of a pair $(M, \mathfrak{A})$ with parity $\vec{p}$ defined by the following relations

$$
\vec{p}(a)=(p(a),-1), \quad \pi=\vec{p}(d)=(0,1), \quad \vec{p}(\omega)=(p(\omega), k) \text { as } \omega \in \Omega_{k} .
$$

A parity for tensor products is defined by ordinary way (by summation).

Consider bivector field $\Theta=\sum_{k} a_{k} b_{k}\left(a_{k}, b_{k} \in \mathfrak{A}\right)$. For the sake of simplicity we put $\vec{p}\left(a_{k}\right)=\vec{p}\left(b_{k}\right)=(0,1)$. We associate with $\Theta$ a bilinear map $\mathcal{H}: \widetilde{\Omega} \rightarrow \widetilde{\Omega} \otimes \mathfrak{A}$

$$
\mathcal{H} \omega=(-1)^{m}\left\{\left[\omega, b_{k}\right] a_{k}-\left[\omega, a_{k}\right] b_{k}\right\} \quad\left(\omega \in \Omega_{m}\right)
$$

Note that

$$
\begin{gathered}
{\left[\left[\omega_{1}, \Theta\right], \omega_{2}\right]=\sum_{k}\left\{\left[\omega_{1}, a_{k}\right]\left[b_{k}, \omega_{2}\right]-\left[\omega_{1}, b_{k}\right]\left[a_{k}, \omega_{2}\right]\right\} \in \widetilde{\Omega}} \\
{\left[\mathcal{H} \omega, \omega_{1}\right]=-(-1)^{\pi p(\omega)}\left[[\omega, \Theta], \omega_{1}\right]}
\end{gathered}
$$

and

$$
d_{\Theta}=\sum_{k}\left\{a_{k} L_{b_{k}}-b_{k} L_{a_{k}}+\left[a_{k}, b_{k}\right]_{d}\right\}
$$

The Poisson brackets $[\cdot, \cdot]_{d_{\Theta}}$ and $\llbracket \cdot, \cdot \rrbracket_{\Theta}$ are defined on $\widetilde{\Omega}$. They obey Leibnitz rule

$$
\begin{aligned}
{\left[\omega, \omega_{1} \omega_{2}\right]_{d_{\Theta}} } & =\left[\omega, \omega_{1}\right]_{d_{\Theta}} \omega_{2}+(-1)^{p\left(\omega_{1}\right)\left(p(\omega)+p\left(d_{\Theta}\right)\right.} \omega_{1}\left[\omega, \omega_{2}\right]_{d_{\Theta}}, \\
\llbracket \omega, \omega_{1} \omega_{2} \rrbracket_{\Theta} & =\llbracket \omega, \omega_{1} \rrbracket_{\Theta} \omega_{2}+(-1)^{p\left(\omega_{1}\right)(p(\omega)+p(\Theta))} \omega_{1} \llbracket \omega, \omega_{2} \rrbracket_{\Theta} .
\end{aligned}
$$

Also they are bound by the relation

$$
\left(d \llbracket \omega_{1}, \omega_{2} \rrbracket_{\Theta}\right)=\left[\left(d \omega_{1}\right),\left(d \omega_{2}\right]_{d_{\Theta}} .\right.
$$

Moreover, as $\omega_{1} \in \widetilde{\Omega}_{m_{1}}, \omega_{2} \in \widetilde{\Omega}_{m_{2}}$

$$
\left[\omega_{1}, \omega_{2}\right]_{d_{\Theta}}=-\left(d\left[\mathcal{H} \omega_{1}, \omega_{2}\right]\right)-(-1)^{m_{1}}\left[\mathcal{H} \omega_{1},\left(d \omega_{2}\right)\right]-(-1)^{m_{1}+m_{2}}\left[\left(d \omega_{1}\right), \mathcal{H} \omega_{2}\right] .
$$

The next theorem follows from the above results. 
TheOrem 8.1. If $\Theta$ is a conditional differential:

$$
\left[d_{\Theta}, d_{\Theta}\right]=0,
$$

then

(i) $\left(\widetilde{\Omega},[\cdot, \cdot]_{d_{\Theta}}, p+\pi\right)$ is a Lie algebra; $d \widetilde{\Omega}$ is a Lie subalgebra and

$$
L^{d_{\ominus}}:\left[\omega_{1}, \omega_{2}\right]_{d_{\Theta}} \mapsto\left[\left[\omega_{1}, d_{\Theta}\right],\left[\omega_{2}, d_{\Theta}\right]\right]
$$

is a homomorphism of Lie algebras;

(ii) $\left(\widetilde{\Omega} / \operatorname{ker} d, \llbracket \cdot, \cdot \rrbracket_{\Theta}, p\right)$ is a Lie algebra and

$$
\mathfrak{L}^{\Theta}: \llbracket \omega_{1}, \omega_{2} \rrbracket_{\Theta} \mapsto\left[\llbracket \omega_{1}, \omega_{2} \rrbracket_{\Theta}, \Theta\right]_{d}=\left[\left[\omega_{1}, \Theta\right]_{d},\left[\omega_{2}, \Theta\right]_{d}\right]
$$

is a homomorphism of Lie algebras.

Proposition 8.2. Relation $[\Theta, \Theta]_{d}=0$ (which is sufficient for (8.1)) holds, if the system of vector fields $\left\{a_{k}, b_{j} \mid a_{k}, b_{j} \in \mathfrak{A}\right\}$ is commutative in $\left(\mathfrak{A}, \llbracket \cdot, \cdot \rrbracket=[\cdot, \cdot]_{d}, p+\pi\right)$ :

$$
\llbracket a_{j}, a_{k} \rrbracket=\llbracket b_{j}, b_{k} \rrbracket=\llbracket a_{j}, b_{k} \rrbracket=0 \quad(\forall j, k) .
$$

The map $\mathcal{H}$ is said to be Hamiltonian, if it is a homomorphism

$$
\begin{aligned}
\mathcal{H}\left(d \llbracket \omega_{1}, \omega_{2} \rrbracket_{\Theta}\right) & =\mathcal{H}\left[\left(d \omega_{1}\right),\left(d \omega_{2}\right)\right]_{d_{\Theta}}=\left[\mathcal{H}\left(d \omega_{1}\right), \mathcal{H}\left(d \omega_{2}\right)\right]_{d}^{\prime} \\
\left(\widetilde{\Omega} / \operatorname{ker} d, \llbracket, \rrbracket_{\Theta}, p\right) & \stackrel{d}{\rightarrow}\left(d \widetilde{\Omega},[,]_{d_{\Theta}}, p+\pi\right) \stackrel{\mathcal{H}}{\rightarrow}\left(\widetilde{\Omega} \otimes \mathfrak{A},[,]_{d}^{\prime}, p^{\prime}\right)
\end{aligned}
$$

TheOREM 8.3. The map $\mathcal{H}$ is Hamiltonian under one of the following conditions:

(i) commutativity conditions are fulfilled

$$
\llbracket a_{j}, a_{k} \rrbracket=\llbracket b_{j}, b_{k} \rrbracket=\llbracket a_{j}, b_{k} \rrbracket=0 \quad(\forall j, k) ;
$$

(ii) $[\Theta, \Theta]_{d}=0$ and $\forall \xi \in \widetilde{\Omega} \otimes \mathfrak{A}:[\xi, d \widetilde{\Omega}]=0$ or $[\xi, d]=0$ implies $\xi=0$;

9. Nambu-Takhtajan algebras. Nambu-Takhtajan algebra structure of order 3 is defined in a linear space $\mathfrak{N}$, if there is defined a 3-linear operation (Nambu bracket) $[\cdot, \cdot, \cdot]: \mathfrak{N} \times \mathfrak{N} \times \mathfrak{N} \rightarrow \mathfrak{N}$ possessing the properties:

(i) skew symmetry

$$
\begin{aligned}
{\left[a_{1}, a_{2}, a_{3}\right] } & =-(-1)^{p_{1} p_{2}}\left[a_{2}, a_{1}, a_{3}\right]= \\
& =-(-1)^{p_{2} p_{3}}\left[a_{1}, a_{3}, a_{2}\right]= \\
& =-(-1)^{p_{1} p_{2}+p_{2} p_{3}+p_{1} p_{3}}\left[a_{3}, a_{2}, a_{1}\right]
\end{aligned}
$$

(ii) fundamental identity: an adjoint action $L_{a_{1}, a_{2}} a_{3}=\left[a_{1}, a_{2}, a_{3}\right]$ is a differentiation with respect to Nambu bracket:

$$
\begin{aligned}
& L_{a_{1}, a_{2}}\left[a_{3}, a_{4}, a_{5}\right]=\left[L_{a_{1}, a_{2}} a_{3}, a_{4}, a_{5}\right]+ \\
& \quad+(-1)^{\left(p_{1}+p_{2}\right) p_{3}}\left[a_{3}, L_{a_{1}, a_{2}} a_{4}, a_{5}\right]+(-1)^{\left(p_{1}+p_{2}\right)\left(p_{3}+p_{4}\right)}\left[a_{3}, a_{4}, L_{a_{1}, a_{2}} a_{5}\right]
\end{aligned}
$$

Here we consider Nambu-Takhtajan algebras only of order 3 . All of results (but not always proves) can be easily transferred to the arbitrary order structure.

One can rewrite (9.1) in the form

$$
\begin{aligned}
{\left[L_{a_{1}, a_{2}}, L_{b_{1}, b_{2}}\right]^{\vee} } & =L_{a_{1}, a_{2}} L_{b_{1}, b_{2}}-(-1)^{\left(p\left(a_{1}\right)+p\left(a_{2}\right)\right)\left(p\left(b_{1}\right)+p\left(b_{2}\right)\right)} L_{b_{1}, b_{2}} L_{a_{1}, a_{2}}= \\
& =L_{\left[a_{1}, a_{2}, b_{1}\right], b_{2}}+(-1)^{p\left(b_{1}\right)\left(p\left(a_{1}\right)+p\left(a_{2}\right)\right)} L_{b_{1},\left[a_{1}, a_{2}, b_{2}\right]}
\end{aligned}
$$


TheOrem 9.1. Consider a linear space $\mathfrak{N}^{(2)}=\mathfrak{N} \otimes \mathfrak{N} / \operatorname{ker} L$. Each of the following formula

$$
\begin{aligned}
{\left[\left(a_{1}, a_{2}\right),\left(b_{1}, b_{2}\right)\right] } & =\left(\left[a_{1}, a_{2}, b_{1}\right], b_{2}\right)+(-1)^{p\left(b_{1}\right)\left(p\left(a_{1}\right)+p\left(a_{2}\right)\right)}\left(b_{1},\left[a_{1}, a_{2}, b_{2}\right]\right), \\
{\left[\left(a_{1}, a_{2}\right),\left(b_{1}, b_{2}\right)\right] } & =-(-1)^{\left(p\left(b_{1}\right)+p\left(b_{2}\right)\right)\left(p\left(a_{1}\right)+p\left(a_{2}\right)\right)}\left(\left[b_{1}, b_{2}, a_{1}\right], a_{2}\right)- \\
& -(-1)^{p\left(a_{1}\right)\left(p\left(b_{1}\right)+p\left(b_{2}\right)\right)}\left(a_{1},\left[b_{1}, b_{2}, a_{2}\right]\right),
\end{aligned}
$$

induces on $\mathfrak{N}^{(2)}$ a Lie algebra structure (the same). In addition $\mathfrak{N}$ becomes a $\mathfrak{N}^{(2)}$-module.

Now we can build the de Rham complex $\Omega(\mathfrak{N}, \mathfrak{N} \otimes \mathfrak{N} /$ ker $L)$. Its elements are skew symmetric polylinear maps of sets of pairs $\left(a_{1}, a_{2}\right)\left(a_{1}, a_{2} \in \mathfrak{N}\right)$. For instance,

$$
\begin{aligned}
(d f)\left(a_{1}, a_{2}\right) & =\left[a_{1}, a_{2}, f\right], \quad(f \in \mathfrak{N}) \\
(d \omega)\left(\left(a_{1}, a_{2}\right),\left(b_{1}, b_{2}\right)\right) & =(-1)^{p(\omega)\left(p\left(a_{1}\right)+p\left(a_{2}\right)\right)}\left[a_{1}, a_{2}, \omega\left(\left(b_{1}, b_{2}\right)\right)\right]- \\
& -(-1)^{\left(p\left(a_{1}\right)+p\left(a_{2}\right)+p(\omega)\right)\left(p\left(b_{1}\right)+p\left(b_{2}\right)\right)}\left[b_{1}, b_{2}, \omega\left(\left(a_{1}, a_{2}\right)\right)\right]- \\
& -\omega\left(\left(\left[a_{1}, a_{2}, b_{1}\right], b_{2}\right)\right)- \\
& -(-1)^{p\left(b_{1}\right)\left(p\left(a_{1}\right)+p\left(a_{2}\right)\right)} \omega\left(\left(b_{1},\left[a_{1}, a_{2}, b_{2}\right]\right)\right)
\end{aligned}
$$

etc.

In such a way one can consider a conditions of a maps to be Hamiltonian leading to a pair of vector fields.

Another variant to introduce a differential geometry structure connected with NambuTakhtajan algebra is as follows. It is well-known that 3-placed bracket generates a parametrically dependent on element of algebra Lie algebra structure with a bracket $[a, b]^{\xi}=$ $[a, \xi, b](\xi \in \mathfrak{N})$.

These brackets satisfy the conditions of Proposition 2.1, and therefore generate a multicomplex $\left(\Omega, d^{\xi}\right)$. Such a multicomplex is an example of $(\mathfrak{N}, \mathfrak{D})$-structure with a commutative space of differentials coinciding with $\mathfrak{N}$.

10. Generalized Jacobians. Let $(\mathfrak{L}, p)$ be a commutative algebra and $D_{1}, D_{2}, D_{3}$ be a commuting differentials of $\mathfrak{L}: D_{j}(a b)=\left(D_{j} a\right) b+(-1)^{q p(a)} a\left(D_{j} b\right)$ (for the sake of simplisity we put $\left.p\left(D_{j}\right)=q\right)$.

The following expression

$$
\begin{aligned}
\left(D_{1}, D_{2},\right. & \left.D_{3}\right)\left(f_{1}, f_{2}, f_{3}\right)=(-1)^{q p_{2}}\left(D_{1} f_{1}\right)\left(D_{2} f_{2}\right)\left(D_{3} f_{3}\right)+(-1)^{q p_{2}}\left(D_{3} f_{1}\right)\left(D_{1} f_{2}\right)\left(D_{2} f_{3}\right)+ \\
& +(-1)^{q p_{2}}\left(D_{2} f_{1}\right)\left(D_{3} f_{2}\right)\left(D_{1} f_{3}\right)-(-1)^{q\left(p_{2}+q\right)}\left(D_{3} f_{1}\right)\left(D_{2} f_{2}\right)\left(D_{1} f_{3}\right)- \\
& -(-1)^{q\left(p_{2}+q\right)}\left(D_{2} f_{1}\right)\left(D_{1} f_{2}\right)\left(D_{3} f_{3}\right)-(-1)^{q\left(p_{2}+q\right)}\left(D_{1} f_{1}\right)\left(D_{3} f_{2}\right)\left(D_{2} f_{3}\right) \quad(10.1)
\end{aligned}
$$

is said to be a generalized Jacobian. We also will write

$$
\left(D_{1}, D_{2}, D_{3}\right)\left(f_{1}, f_{2}, f_{3}\right)=\left|\begin{array}{ccc}
D_{1} f_{1} & D_{1} f_{2} & D_{1} f_{3} \\
D_{2} f_{1} & D_{2} f_{2} & D_{2} f_{3} \\
D_{3} f_{1} & D_{3} f_{2} & D_{3} f_{3}
\end{array}\right|=\left|\begin{array}{lll}
D_{k} f_{1} & D_{k} f_{2} & D_{k} f_{3}
\end{array}\right|
$$


Proposition 10.1. Operation (10.1) possesses the following properties:

(i) skew symmetry

$$
\begin{aligned}
\left(D_{1}, D_{2}, D_{3}\right)\left(f_{1}, f_{2}, f_{3}\right) & =-(-1)^{p_{1} p_{2}}\left(D_{1}, D_{2}, D_{3}\right)\left(f_{2}, f_{1}, f_{3}\right)= \\
& \left.=-(-1)^{(} D_{1}, D_{2}, D_{3}\right)\left(f_{1}, f_{3}, f_{2}\right)= \\
& =-(-1)^{p_{1} p_{2}+p_{2} p_{3}+p_{1} p_{3}}\left(D_{1}, D_{2}, D_{3}\right)\left(f_{1}, f_{2}, f_{3}\right)
\end{aligned}
$$

(ii) differentiation with respect to the multiplication in algebra $(\mathfrak{L}, p)$ :

$$
\begin{aligned}
\left(D_{1}, D_{2}, D_{3}\right)\left(f_{1} \cdot g, f_{2}, f_{3}\right) & =(-1)^{p_{1} q} f_{1} \cdot\left(D_{1}, D_{2}, D_{3}\right)\left(g, f_{2}, f_{3}\right)+ \\
& +(-1)^{p(g)\left(p_{1}+q\right)} g \cdot\left(D_{1}, D_{2}, D_{3}\right)\left(f_{1}, f_{2}, f_{3}\right)
\end{aligned}
$$

Introduce an action $L_{f_{1}, f_{2}}^{D_{1}, D_{2}, D_{3}} f_{3}=\left(D_{1}, D_{2}, D_{3}\right)\left(f_{1}, f_{2}, f_{3}\right)$. It is a differentiation with respect to the multiplication in $(\mathfrak{L}, p)$ :

$$
L_{f_{1}, f_{2}}^{D_{1}, D_{2}, D_{3}}(f \cdot g)=\left(L_{f_{1}, f_{2}}^{D_{1}, D_{2}, D_{3}} f\right) \cdot g+(-1)^{p(f) p(g)}\left(L_{f_{1}, f_{2}}^{D_{1}, D_{2}, D_{3}} g\right) \cdot f .
$$

THEOREM 10.2. Expression (10.1) defines a Nambu-Takhtajan structure $(\mathfrak{L},[\cdot, \cdot, \cdot], p)$ in $\mathfrak{L}$ with the bracket:

$$
\left[f_{1}, f_{2}, f_{3}\right]=\left(D_{1}, D_{2}, D_{3}\right)\left(f_{1}, f_{2}, f_{3}\right)
$$

Note that it is evident for any differentiation $D$ of algebra $\mathfrak{L}$ that

$$
\begin{array}{rllll}
D\left|D_{k} f_{1} \quad D_{k} f_{2} \quad D_{k} f_{3}\right| & =\left|\begin{array}{llll}
D D_{k} f_{1} & D_{k} f_{2} & D_{k} f_{3}
\end{array}\right|+ \\
& +\left|\begin{array}{llll}
D_{k} f_{1} & (-1)^{p(D)\left(p_{1}+q\right)} D D_{k} f_{2} & D_{k} f_{3}
\end{array}\right|+ \\
& +\left|\begin{array}{llll}
D_{k} f_{1} & D_{k} f_{2} & (-1)^{p(D)\left(p_{1}+p_{2}\right)} D D_{k} f_{3}
\end{array}\right|
\end{array}
$$

This is an analog of a differentiation rule for a determinant.

It remains to check that for $D=L_{g_{1}, g_{2}}^{D_{1}, D_{2}, D_{3}}$ one can interchange $D$ and $D_{k}$ in this formula, i. e.

$$
\begin{aligned}
D\left|D_{k} f_{1} \quad D_{k} f_{2} \quad D_{k} f_{3}\right| & =\left|\begin{array}{lll}
(-1)^{p(D) q} D_{k} D f_{1} & D_{k} f_{2} & D_{k} f_{3}
\end{array}\right|+ \\
& +\left|\begin{array}{llll}
D_{k} f_{1} & (-1)^{p(D) p_{1}} D_{k} D f_{2} & D_{k} f_{3}
\end{array}\right|+ \\
& +\left|\begin{array}{llll}
D_{k} f_{1} & D_{k} f_{2} & (-1)^{p(D)\left(p_{1}+p_{2}+q\right)} D_{k} D f_{3}
\end{array}\right|
\end{aligned}
$$

This formula means that an adjoint action of Nambu bracket is a differentiation with respect to the operation defined by Namby bracket itself. And this fact is equivalent to fundamental identity.

(10.3) follows from (10.2) using an auxiliary relation

$$
(-1)^{\left(p_{3}+q\right) p_{5}}\left|\left(D_{1}, D_{2}, D_{3}\right)\left(D_{k} f_{1}, f_{2}, f_{3}\right) \quad D_{k} f_{4} \quad D_{k} f_{5}\right|+\stackrel{3,4,5}{\mathfrak{y}}=0,
$$

(by the sign $\mathfrak{y}$ we denote terms which are obtained from the latter one by the cyclic permutation of indexes mentioned).

To check it we use direct laborious computation. (For Nambu-Takhtajan algebra of order $n>3$ such a direct computation becomes badly visible).

Consider an examples. In the case $p \equiv 0$ (10.1) is an ordinary Jacobian. We are interesting in a common case. 
Let $\Omega(M, \mathfrak{A})$ be a complex. For instance, let $\mathfrak{L}=\Omega$ be an exterior algebra of differential forms. To build a generalized Jacobian one need to select a system of commuting differentials. We propose two different ways to do this:

(i) $D_{j}=i_{a_{j}}\left(a_{j} \in \mathfrak{A}, j=1,2,3\right)$;

(ii) $D_{j}=d^{\xi_{j}}\left(\xi_{j} \in \mathfrak{A}, j=1,2,3\right)$ for multicomplex (i. e. the space of differentials $\mathfrak{D}$ acts in $\widetilde{\Omega}$ ).

\section{References}

[1] I. M. Gelfand and Yu. L. Daletsky, Lie Superalgebras and Hamiltonian Operators. Rep. No. 16 Sem. Supermanifolds, Dept. Math. Univ. Stockholm, 1987, 26 p.

[2] I. M. Gelfand, Yu. L. Daletskii and B. L. Tsygan, On a Variant of Non-Commutative Differential Geometry. Soviet Math. Dokl. 40 (1990), 2, 422-426.

[3] I. M. Gelfand and I. Ya. Dorfman, Hamiltonian operators and algebraic structures connected with them. Funct. anal. appl. 13 (1979), 4, 13-30.

[4] I. M. Gelfand and I. Ya. Dorfman, Hamiltonian operators and infinite dimensional Lie algebras. Funct. anal. appl. 15 (1981), 3, 23-40.

[5] M. Dubois-Violette, R. Kerner and J. Madore, Noncommutative Differential Geometry of Matrix Algebras. J. Math. Phys. 31 (1990), 2, 316-322.

[6] Yu. L. Daletskii and B. L. Tsygan, Operations on Hochschild and Cyclic Complexes. $K$-Theorie, (in print).

[7] Yu. L. Daletskii and B. L. Tsygan, Hamoltonian Operators and Hochschild Homology. Funct. anal. appl. 19 (1985), 4, 82-83.

[8] A. Cabras and A. M. Vinogradov, Extension of the Poisson Bracket to Differential Forms and Multi-Vector Fields. J. Geom. and Physics, 9 (1992), 75-100.

[9] Yu. L. Daletskii and V. A. Kushnirevitch, Poisson and Nijenhuis Brackets for Differential Forms on Non-Commutative Manifold. SFB 237 - Preprint Nr 274, Institut für Mathemetik, Ruhr-Universität-Bochum, September, 1995. 29 p.

[10] Y. Nambu, Generalized Hamiltonian Dynamics. Phys. Review D7 (1973), 8, 2405-2412.

[11] L. Takhtajan, On Foundation of the Generalized Nambu Mechanics. Commun. Math. Phys. 160 (1994), 295-315.

[12] L. Takhtajan, Higher Order Analog of Chevalley-Eilenberg Complex and Deformation theory of $N$-gebras. Algebra and Analysis, 6 (1994), 2, 262-272.

[13] Yu. L. Daletskii, Hamiltonian Operators in Graded Formal Calculus of Variations. Funct. anal. appl. 20 (1986), 2, 62-64.

[14] B. A. Kupershmidt, Elements of Superintegrable Systems. Reidel, 1987.

[15] A. Connes, Géométrie Non Commutative. InterEditions, 1990.

[16] S. L. Woronowicz, Differential Calculus on Compact Matrix Pseudogroups (Quantum Groups). Commun. Math. Phys. 122 (1989), 125-170. 